\title{
Significance of immunoglobulin deposition in peripheral nerve in neuropathies associated with paraproteinaemia
}

\author{
M. S W A S H, J.PERR I N, A N D M. S. S C H W A R T Z \\ From the Departments of Clinical Immunology and Pathology, The London Hospital Medical College, \\ and the Department of Neurology, The London Hospital, London
}

SUMMARY Direct and indirect immunofluorescent studies of sural nerves were carried out in two patients with paraproteinaemia and neuropathy, in four other patients with axonal or demyelinating neuropathies, and in one normal sural nerve. IgM was demonstrated directly in the two cases of paraproteinaemia and neuropathy, and indirectly, using the serum of one of these cases, in a case of axonal neuropathy and in one case of chronic Guillain-Barré syndrome. In the latter case, IgM deposition also occurred after exposure to normal serum. These results suggest that the paraprotein itself did not directly cause neuropathy, but that immunoglobulin deposition is probably a secondary process, caused by diffusion into damaged nerves.

Peripheral neuropathy occurs in association with paraproteinaemias of various causes (McLeod and Walsh, 1975; Read et al., 1978) but the pathogenesis of these neuropathies is obscure. In this paper we report experiments on the immunofluorescent binding of the paraprotein from two patients with paraproteinaemia and neuropathy to their own sural nerves, and to the sural nerves of five other patients, four with demyelinating or axonal neuropathies not associated with paraproteinaemias, and one without neuropathy or paraproteinaemia. Immunofluorescent binding of normal serum to these nerves was also studied.

\section{Case reports}

CASE 1

A 66 year old man presented with weakness and tingling in his legs. A chest radiograph in May 1976 revealed a small lobulated opacity in the right lower lobe but bronchoscopy and sputum cytology were both negative. The ankle jerks were absent, and there was a mild distal impairment of sensation, with a slightly ataxic gait. In May 1977 he was reassessed because of increasing difficulty with walking. There was slight weakness of the intrinsic muscles of both hands, with impaired two-

Address for correspondence and reprint requests: Dr M. Swash, Department of Neurology, The London Hospital, London, E1 1BB. Accepted 12 September 1978 point discrimination in the fingers; in the legs there was distal weakness and sensory impairment. The ankle jerks were absent but the other reflexes were just present. The left median motor nerve conduction velocity was $19 \mathrm{~m} / \mathrm{s}$, and an $F$ wave study revealed a proximal conduction velocity of $25 \mathrm{~m} / \mathrm{s}$. The left sural sensory nerve action potential was absent. The chest radiograph showed no change in the small lesion in the right lower lobe. The ESR was $65 \mathrm{~mm} / \mathrm{hr}$. Serum protein electrophoresis showed a paraprotein. Immunoelectrophoresis revealed an IgA level of $1.7 \mathrm{~g} / 1$ (normal $1.7-4.1 \mathrm{~g} / \mathrm{l}$ ), IgG $11.0 \mathrm{~g} / 1$ (normal 6.0-12.0 g/1), and IgM $9.0 \mathrm{~g} / 1$ (normal $0.6-1.8 \mathrm{~g} / \mathrm{l}$ ). The paraprotein consisted of IgM heavy chains and kappa light chains, and the urine contained Bence-Jones protein, which consisted of kappa light chains. A mitochondrial antibody was found in the patient's serum. This mitochondrial antibody was found in the $\operatorname{IgA}$ and $\operatorname{IgG}$ fractions, but not in the $\operatorname{IgM}$ kappa paraprotein. Marrow biopsy and a radiographic bone survey showed no evidence of multiple myelomatosis. Sural nerve biopsy was performed. In May 1978, repeat bronchoscopy showed poorly differentiated squamous cell carcinoma.

\section{CASE 2}

For a year this 69 year old man had noticed progressive distal numbness and paraesthesiae. During this time his walking had deteriorated. On exam- 
ination there was a glove and stocking sensory disturbance with distal weakness. The tendon reflexes were absent. The ESR was $75 \mathrm{~mm} / \mathrm{hr}$ and the CSF protein was $1.4 \mathrm{~g} / 1$. The right median motor nerve conduction velocity was $30 \mathrm{~m} / \mathrm{s}$. Median and sural nerve sensory action potentials could not be obtained. Serum protein electrophoresis showed increase in gamma globulins with an IgM level of $4.0 \mathrm{~g} / 1$. This IgM was polyclonal. Sural nerve biopsy was performed.

\section{Methods}

The sural nerve biopsy samples were embedded in Araldite and prepared for light and electron microscopy. In addition, small pieces of these specimens were snap frozen in isopentane/liquid nitrogen. It was thus possible to study frozen sections of peripheral nerve from the two cases of neuropathy associated with paraproteinaemia (cases 1 and 2), and to compare their immunofluorescent reactions with nerve biopsy samples from two cases of Guillain-Barré syndrome (cases 3 and 4) and cases of idiopathic axonal neuropathy (case 5) and Déjerine-Sottas disease (case 6). A normal sural nerve (case 7) was obtained at necropsy from a patient who had died of myocardial infarction (Table 1).

\section{IMMUNOFLUORESCENCE}

Cryostat sections $6 \mu \mathrm{m}$ thick, were stained by the direct immunofluorescent technique, using sheep antihuman fluorescent-labelled conjugates to IgG, $\operatorname{IgM}, \operatorname{IgA}$, and rabbit antihuman conjugates to $\mathrm{C} 3$, fibrin, and albumin. In separate experiments cryostat sections of these nerves were first exposed to case 1 serum or to a normal serum and stained by the indirect immunofluorescent technique using sheep or rabbit antihuman IgM fluorescent conjugate, as in the direct technique. The stained sections were examined by ultraviolet epi-illumination using a Zeiss universal research microscope.

In case 1 the IgM fraction was separated on a Sephadex G200 chromatograph column: 7S IgM was identified by the method of Stobo and Tommasi (1967).

\section{Results}

\section{NERVE PATHOLOGY}

In case 1 there was a marked loss of myelinated fibres (Fig. 1). Many large axons were devoid of myelin, and others had abnormally thin myelin sheaths. Remaining myelin rings were often distorted or reduplicated. No inflammatory infiltrates or amyloid deposits were seen. Electron micro-

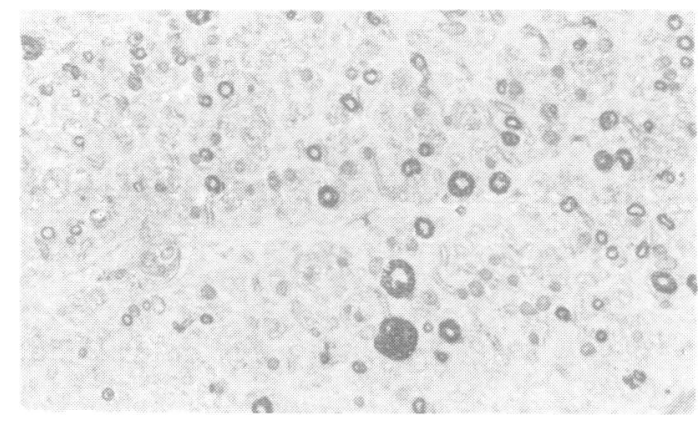

Fig. 1 Case 1. Sural nerve transverse section.

Toluidine blue, original magnification $\times 560$. Few myelin rings remain: some are thinner than normal. Unmyelinated large axons are present.

scopy showed degenerative changes in some myelin rings. There were increased amounts of endoneurial collagen, and the perineurium was slightly thickened. Onion bulbs were not present, and unmyelinated nerve fibres were normal. These appearances were consistent with a neuropathy of predominantly demyelinating type.

In case 2 there were similar abnormalities. No inflammatory infiltrates or amyloid deposits were seen.

Cases 3 and 4 showed the typical features of chronic Guillain-Barré syndrome including segmental demyelination with sparse inflammatoryo cell infiltrates, loss of myelin rings, and onion bulbo formation. In case 5 there was axonal loss with regenerating axonal clusters. Remaining fibres were normally myelinated. In case 6 there was a severe demyelinating neuropathy with prominent onionbulb formation. There was no amyloid. In case 7 the sural nerve was normal.

\section{IMMUNOFLUORESCENCE}

Direct immunofluorescence

In cases 1 and 2 there was positive staining for IgM in the myelin sheaths of the degenerating nerve. The staining, most clearly identified in the cross section of the nerve, formed a collar in the thickness of the myelin sheath round the central, unstained axis cylinder (Fig. 2). The IgM deposited in the myelin had the same kappa specificity as the serum paraprotein and as the urinary Bence-Jones protein (Table 1). In case 2 , although the serum IgM was polyclonal, with a mixture of kappa and lambda light chains, there was no staining of the myelin sheaths in the sural nerve biopsy specimen with either anti-kappa or anti-lambda conjugates. The IgM staining in this case was much weaker than in case 1 , with less deposition of the immunoglobulin, and this probably accounts for the nega- 

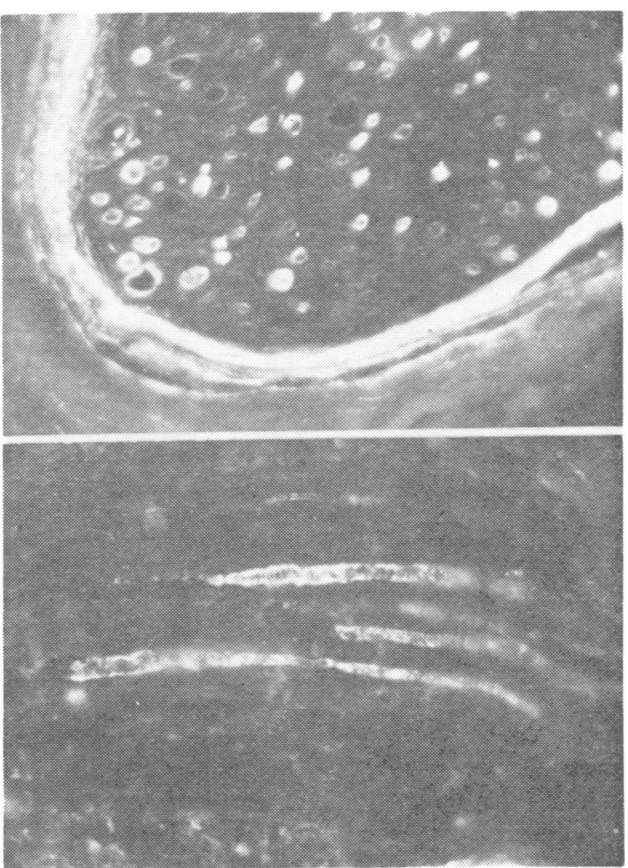

Fig. 2 Case 1. Direct immunofluorescence for IgM in sural nerve, original magnification $\times 320$. The perineurium and the whole thickness of the remaining myelin sheaths show fluorescent staining. Transverse and longitudinal sections.

tive reaction for kappa and lambda light chain components. The remaining cases showed no immunoglobulin deposition in the myelin sheaths or elsewhere in the nerve (Table 1). No nerve showed detectable deposition of $\mathrm{C} 3$, albumin, or fibrin.

\section{Indirect immunofluorescence}

To ascertain if the IgM-kappa paraprotein in case 1 had any affinity for normal or abnormal nerve, a number of the sural nerve samples were exposed in vitro to serum from case 1 , and to a control serum with a normal level of polyclonal IgM (see Table 2). In case 4 , one of the two patients with Guillain-Barré syndrome, there was positive staining of both myelin and perineurium after treatment with both case 1 serum (Fig. 3), and normal serum (Fig. 4). Staining was more intense with case 1 serum. In the other case of Guillain-Barré syndrome (case 3 ) there was no staining of the nerve by either case 1 or normal serum. In case 5 , an axonal neuropathy, there was also positive staining after treatment with case 1 serum, but not after normal serum. Indirect staining in cases 4 and 5, was patchy, and appeared to be located superficially on the myelin sheath (Figs. 2 and 3 ) in contrast to the denser, full thickness staining seen by direct immunofluorescence in case 1 (Fig. 1).

Table 2 Results of fluorescent staining for IgM in biopsy treated with case 1 serum, and normal serum specimens

\begin{tabular}{llll}
\hline $\begin{array}{l}\text { Case } \\
\text { number }\end{array}$ & $\begin{array}{l}\text { Case 1 } \\
\text { serum } \\
\text { IgM-kappa }\end{array}$ & $\begin{array}{l}\text { Normal } \\
\text { serum } \\
\text { polyclonal IgM }\end{array}$ & $\begin{array}{l}\text { Control } \\
\text { (anti-IgM) } \\
\text { (alone) }\end{array}$ \\
\hline 3 & - & - & - \\
4 & + & + & - \\
5 & + & - & - \\
6 & - & - & - \\
Normal & - & - & - \\
nerve & & & \\
\hline
\end{tabular}

Table 1 Results of direct immunofluorescent staining of sural nerve biopsies

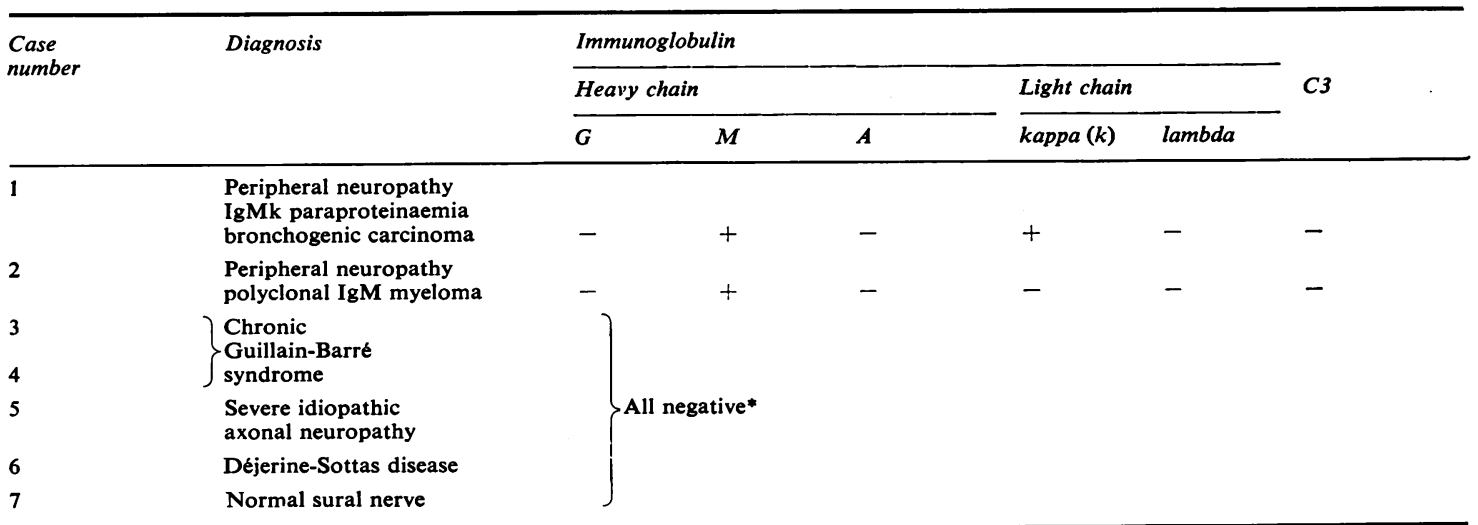

*Fluorescent stains for fibrin and albumin were negative in all these cases. 

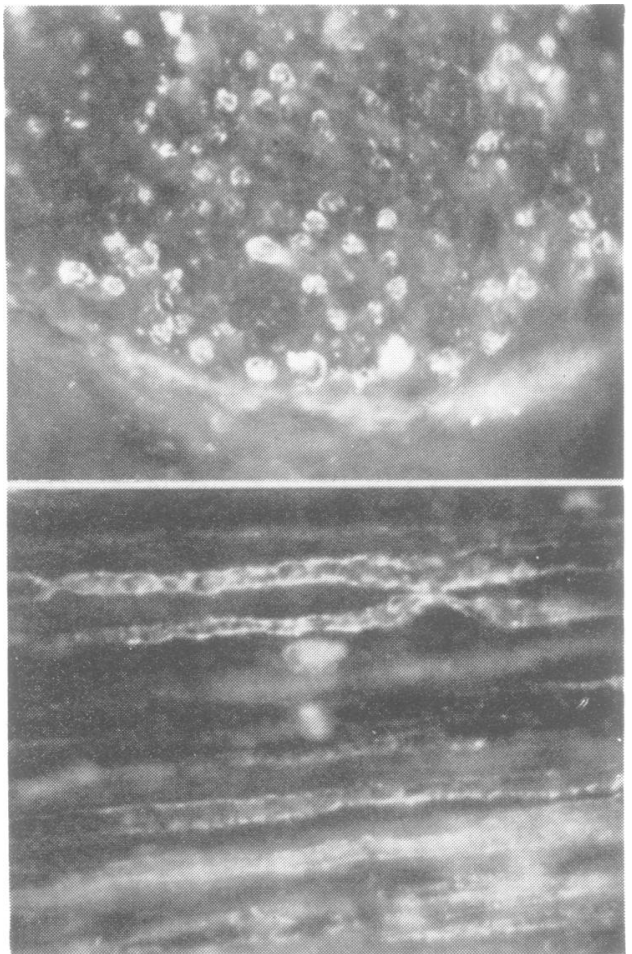

Fig. 3 Case 4. Chronic Guillain-Barré syndrome. Transverse and longitudinal sections, original magnification $\times 450$. Indirect immunofluorescence for IgM in sural nerve after exposure to case 1 serum. There is fluorescence in perineurium, and on the surface of the remaining myelin sheaths.

\section{Discussion}

IgM was detected in the myelin sheaths and perineurium of the sural nerve specimens by direct immunofluorescence in both the patients (cases 1 and 2) with peripheral neuropathy and paraproteinaemia. In case 1 this IgM was the same kappaspecific monoclonal paraprotein present in the serum, and in the urine. In case $2 \operatorname{IgM}$ was deposited in insufficient amounts to allow identification of its light chain polyclonality. In previous reports monoclonal IgM-kappa has been demonstrated by the direct immunofluorescent technique in the myelin sheath (Propp et al., 1975; Hobbs et al., 1975), and in the endoneurium and perineurium (Chazot et al., 1974, 1976) in patients with Waldenström's macroglobulinaemia and peripheral neuropathy. In addition, monoclonal IgG and $\mathrm{IgA}$ has been found in nerve biopsy samples of patients with myeloma and peripheral neuropathy (Chazot et al. 1976). However, peripheral neuropathy is an

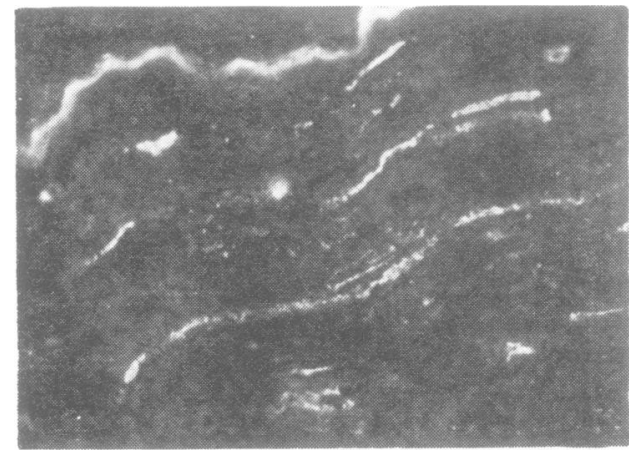

Fig. 4 Case 4. Chronic Guillain-Barré syndrome. Longitudinal section, original magnification $\times 450$. Indirect immunofluorescence for $\operatorname{IgM}$ in sural nerve after exposure to normal serum. There is faint staining of myelin sheaths.

uncommon complication of paraproteinaemia. Chazot et al. (1976) found an incidence of only $9.8 \%$ in a series of 1602 patients with myeloma, Waldenström's macroglobulinaemia, and benign paraproteinaemia, and the significance of immunoglobulin binding to nerve in such cases is, therefore, uncertain.

The presence of immunoglobulin bound to peripheral nerve might indicate a specific immunological reaction against antigen in the myelin sheath, endoneurium, or perineurium. This seems unlikely, however, since these structures differ antigenically. Moreover, practically all autoantibodies in sustained autoimmune responses are found in several immunoglobulin classes. For example, in case 1, a mitochondrial antibody was found in the $\operatorname{Ig} \mathrm{A}$ and $\operatorname{IgG}$ fractions, but not in the $\operatorname{IgM}$ fraction-that is, the autoantibody was polyclonal in respect of heavy chain class. On the other hand. monoclonal paraproteins have recently been shown to have identifiable antibody specificities of haptenic type (Seligmann and Brouet, 1973; Potter, 1977). Another indication of possible specific antibody activity in tissue-deposited protein is the concomitant demonstration of complement, but no $\mathrm{C} 3$ was detected in either case 1 or case 2 .

Van Lis and Jennekens (1977) showed that several plasma proteins, including immunoglobulins, could be identified in the epineurium, perineurium, and endoneurium of both abnormal and normal sural nerve biopsy samples. The distribution varied according to the pathology, and the molecular weight of the protein. They suggested that endoneurial blood vessels function as a barrier with graded permeability. In pathological conditions protein leakage might be increased, and reabsorption from nerves to vessels interrupted. 
Olsson (1968) demonstrated abnormal permeability to albumin in peripheral nerves, particularly across endoneurial blood vessels, in rats treated with isoniazid for two weeks, suggesting that this neuropathy is associated with changes in the bloodnerve barrier. This mechanism might account for deposition of $\operatorname{IgM}$ in the abnormal nerves of our cases 1 and 2 . Indeed, in our case 1 the neuropathy was predominantly demyelinating, and was associated not only with paraproteinaemia, but with carcinoma of the bronchus. The neuropathy associated with paraproteinaemia in myeloma and Waldenström's disease is typically axonal in type (McLeod and Walsh, 1975) although demyelinating neuropathy has rarely been reported with multiple myeloma (Mayo et al., 1965). In case 2 the EMG and pathological findings were more typical of the axonal neuropathy usually associated with paraproteinaemia.

Our indirect immunofluorescent studies (Table 2) indicate that immunoglobulins are deposited, or develop affinity for peripheral nerve components, as a secondary phenomenon in nerves rendered susceptible by particular types of damage. A nerve from one of the patients (case 4) with chronic Guillain-Barré syndrome (see Luitjen and Baart de la Faille-Kuyper, 1972), and the nerve from case 5 , with an axonal neuropathy, both showed affinity for the monoclonal IgM-Kappa in case 1 serum. The other cases, and a normal nerve, as also shown by Propp et al. (1975), showed no such affinity. None of these nerves showed direct immunofluorescent staining (Table 1). Further, in case 4 indirect staining was present after exposure of the nerve specimen to normal serum. These experiments indicate an affinity of IgM (from case 1 serum) for myelin in both demyelinating and axonal neuropathies, suggesting that this affinity is not a specific autoimmune response. Indeed Carter (1977) found that 39 of 63 IgM paraproteins showed affinity for a wide range of axonal and glial antigens, but no such affinity was found in over $150 \mathrm{IgG}$ or IgA paraproteins, or in patients with increased levels of polyclonal IgM.

We, therefore, suggest that there is no direct or causal relation between neuropathy and paraproteinaemia, but that the neuropathy is an associated feature of the underlying disorder, similar to the neuropathy associated with malignant disease. Immunoglobulin (IgM) deposition is probably a secondary process, accounted for by diffusion into damaged nerves, and by affinity between the IgM and peripheral nerve components, especially myelin sheath and perineurium.

\section{References}

Carter, P. M. (1977). Monoclonal proteins. In Immunology in Medicine, p. 980 . Edited by E. J. Holborow and W. G. Reeves. Academic Press: London. Chazot, G., Berger, G., Bady, B., Dumas, R. Creysel, R., Tommasi, M., Schott, B., and Girard, P. (1974). Neuropathie periphérique au cours des dysglobulinémies malignes: aspects immunopathologiques. $\mathbf{L a}$ Nouvelle Presse Medicale, 3, 1355-1358.

Chazot, G., Berger, B., Carrier, H., Barboret, C., Bady, B., Dumas, R., Creysel, R., and Schott, B. (1976). Manifestations neurologiques des gammopathies monoclonales. Revue Neurologique, 132, 195-212.

Hobbs, J. R., Carter, P. M., Cooke, K. B., Foster, M., and Oon C-J. (1975). IgM paraproteins. Journal of Clinical Pathology, 28, Supplement 6, 54-64.

Luitjen, J. A. F. M., and Baart de la Faille-Kuyper, E. H. (1972). The occurrence of IgM and complement factors along myelin sheaths of peripheral nervesan immunohistochemical study of the Guillain-Barré syndrome. Journal of the Neurological Sciences, 15, 219-224.

Mayo, C. A., Daniels, A., and Barron, K. D. (1965). Polyneuropathy in the osteosclerotic form of multiple myeloma. Transactions of the American Neurological Association, 93, 240.

McLeod, J. G., and Walsh, J. C. (1975). Neuropathies associated with paraproteinaemias and dysproteinaemias. In Peripheral Neuropathy, vol. 2, pp. 10121029. Edited by P. J. Dyck, P. K. Thomas, and E. H. Lambert. W. B. Saunders: Philadelphia.

Olsson, Y. (1968). Studies on vascular permeability in peripheral nerves 3 Permeability changes in vasa nervorum and exudation of serum albumin in INHinduced neuropathy in the rat. Acta Neuropathologica (Berlin), 11, 103-112.

Potter, M. (1977). Antigen-binding myeloma proteins of mice. In Advances in Immunology, vol. 25, pp. 141-211. Edited by H. G. Kunkel and F. J. Dixon. Academic Press: London.

Propp, R. P., Means, E., Deibel, R., Sherer, G., and Barron, K. (1975). Waldenström's macroglobulinemia and neuropathy: deposition of M-component on myelin sheaths. Neurology (Minneapolis), 25, 980-988.

Read, D. J., Vanhegan, R. I., and Matthews, W. B. (1978). Peripheral neuropathy and benign IgG paraproteinaemia. Journal of Neurology, Neurosurgery, and Psychiatry, 41, 215-219.

Seligmann, M., and Brouet, J. C. (1973). Antibody activity of human myeloma globulins. Seminars in Hematology, 10, 163-177.

Stobo, J. D., and Tommasi, T. B. (1967). A low molecular weight immunoglobulin antigenically related in 19S IgM. Journal of Clinical Investigation, 46, 1329-1337.

Van Lis J. M. J., and Jennekens, F. G. I. (1977). Plasma proteins in human peripheral nerve. Journal of the Neurological Sciences, 34, 329-341. 